\title{
MODIS and VIIRS Lunar Observations and Applications
}

\author{
Xiaoxiong Xiong ${ }^{1}$, Zhipeng Wang ${ }^{2}$, Junqiang Sun ${ }^{2}$, Amit Angal ${ }^{3}$, Jon Fulbright ${ }^{2}$, and James Butler ${ }^{1}$ \\ ${ }^{1}$ Sciences and Exploration Directorate, NASA/GSFC, Greenbelt, MD 20771 \\ ${ }^{2}$ Sigma Space Corp., 4801 Forbes Boulevard, Lanham, MD 20706 \\ ${ }^{3}$ Science Systems and Applications Inc., 10210 Greenbelt Road, Lanham, MD 20706
}

\begin{abstract}
Terra and Aqua MODIS have successfully operated for more than 13 and 11 years since their launch in 1999 and 2002, respectively. The VIIRS instrument on-board the S-NPP launched in 2011 has also operated for nearly 2 years. Both MODIS and VIIRS make observations in the reflective solar and thermal emissive regions and their on-orbit calibration and characterization are provided by a set of on-board calibrators (OBC). In addition, lunar observations have been made on a regular basis to support sensor on-orbit calibration. This paper provides a brief overview of MODIS and VIIRS instrument on-orbit calibration and characterization activities. It describes the approaches and strategies developed to schedule and perform on-orbit lunar observations. Specific applications of MODIS and VIIRS lunar observations discussed in this paper include radiometric calibration stability monitoring and performance assessment of sensor spatial characterization. Results derived from lunar observations, such as sensor response (or gain) trending and band-to-band registration, are compared with that derived from sensor OBC. The methodologies and applications presented in this paper can also be applied to other earth observing sensors.
\end{abstract}

Keywords: MODIS, VIIRS, radiometric calibration, spatial characterization, BBR, MTF, lunar observations

\section{INTRODUCTION}

The Moderate Resolution Imaging Spectroradiometer (MODIS) shown in Figure 1(a) is a key instrument for the NASA's Earth Observing System (EOS) Terra and Aqua missions. Since launch, Terra and Aqua MODIS have successfully operated for more than 13 and 11 years, respectively. MODIS is a scanning radiometer using a two-sided scan mirror and collects data in 36 spectral bands, covering wavelengths from 0.41 to $14.5 \mu \mathrm{m}$. MODIS observations are made in 3 spatial resolutions (nadir): $250 \mathrm{~m}$ (bands 1-2), $500 \mathrm{~m}$ (bands 3-7), and $1 \mathrm{~km}$ (bands 8-36). It carries a set of onboard calibrators (OBC), including a solar diffuser (SD), a solar diffuser stability monitor (SDSM), a blackbody (BB), and a spectroradiometric calibration assembly (SRCA). Nearly 40 science data products have been routinely generated from MODIS observations, covering applications and studies of the earth's land, ocean, and atmospheric properties ${ }^{1-4}$.

The Visible/Infrared Imager Radiometer Suite (VIIRS) sensor shown in Figure 1(b) was designed and developed with a strong MODIS heritage and built by the same instrument vendor. Its OBC include MODIS-like SD, SDSM, and BB. VIIRS does not have an on-board device like MODIS SRCA. Instead of a scan mirror, VIIRS uses an imaging system that includes a rotating telescope assembly (RTA) and a half-angle mirror (HAM). The first VIIRS is currently operated on-board the Suomi National Polar-orbiting Partnership (S-NPP) spacecraft launched in October 2011. The next VIIRS instrument is currently being built and tested at the instrument vendor facility and will be operated on-board the Joint

Sensors, Systems, and Next-Generation Satellites XVII, edited by Roland Meynart, Steven P. Neeck, Haruhisa Shimoda, Proc. of SPIE Vol. 8889, 88890V · (C) 2013 SPIE · CCC code: 0277-786X/13/\$18 · doi: 10.1117/12.2028954 
Polar Satellite System (JPSS) satellite. It is currently scheduled for a launch in 2017. VIIRS observations are made in 22 spectral bands covering wavelengths from 0.41 to $12.5 \mu \mathrm{m}$. Among them are 16 moderate (M) resolution bands with a nadir spatial resolution of $750 \mathrm{~m}, 5$ imaging (I) bands at $375 \mathrm{~m}$ nadir spatial resolution, and a day/night band (DNB) at a nadir spatial resolution of $750 \mathrm{~m}$. Seven of the moderate resolution bands (M1-M5, M7, and M13) are the dual gain bands, which can make measurements over a large dynamic range. The Sensor Data Records (SDR) derived from VIIRS observations are used for the production of its Environmental Data Records (EDR). VIIRS observations have enabled some new applications, such as those derived from the DNB observations ${ }^{5-8}$.

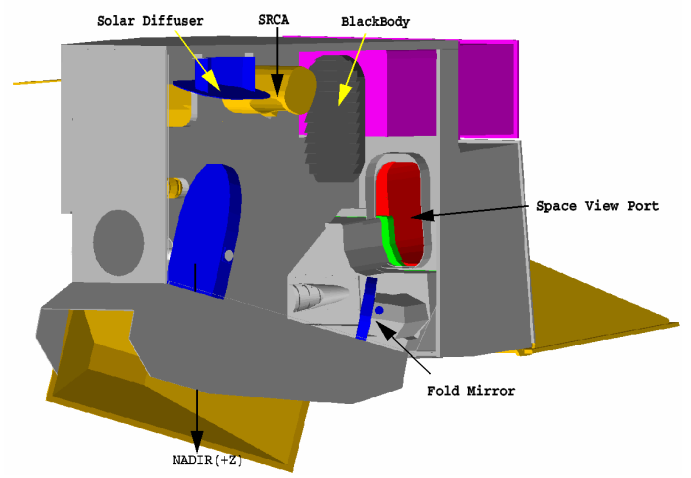

(a)

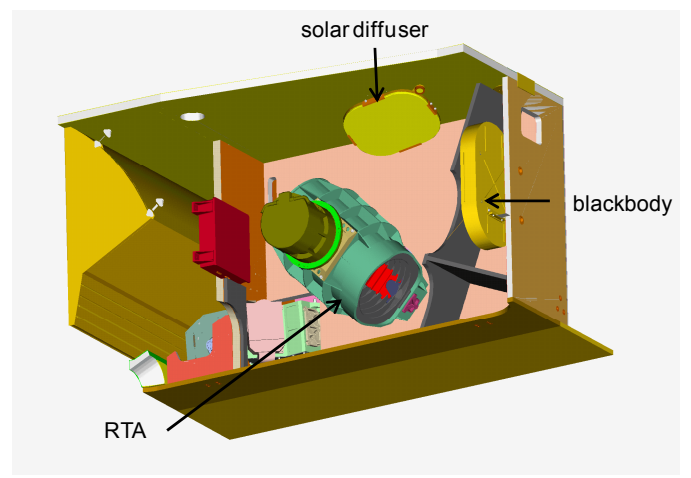

(b)

Figure 1 MODIS (left) and VIIRS (right) instruments and their on-board calibrators (OBC)

In addition to the sensor OBC, lunar observations have been scheduled and made on a regular basis for both Terra and Aqua MODIS, as well as for the S-NPP VIIRS. Following a brief description of MODIS and VIIRS on-board calibration activities, this paper discusses their lunar observations and various applications, including lunar calibration scheduling and implementation strategies and the methodologies developed to use the Moon for sensor radiometric calibration stability monitoring and spatial performance assessment. Results derived from MODIS and VIIRS lunar observations, such as sensor response (or gain) trending, band-to-band registration (BBR), and modulation transfer function (MTF), are also presented and compared, whenever possible, with that derived from the $\mathrm{OBC}$ and other approaches. It is demonstrated in this paper that lunar observations have played and will continue to play an important role in support of sensor on-orbit calibration and characterization. It is expected that the methodologies developed and presented in the paper and various lessons learned can be easily adopted and applied to other earth observing sensors.

\section{MODIS AND VIIRS ON-BOARD CALIBRATION}

MODIS bands 1-19 and 26 cover wavelengths from 0.41 to $2.3 \mu \mathrm{m}$ and are referred to as the reflective solar bands (RSB). They are calibrated on-orbit by a SD and SDSM system. The SD bi-directional reflectance factor (BRF) was characterized pre-launch and its on-orbit changes are tracked by the SDSM. In general, the SD and SDSM calibration is scheduled via command uploads to the spacecraft on a weekly basis at the mission beginning and then on a bi-weekly basis. As mission has continued and operated beyond the sensor specified design lifetime (6 years), the SD/SDSM calibration frequencies have been reduced further. If no SD/SDSM calibration is scheduled, the SD door is designed to be in a close position. MODIS bands 20-25 and 27-36 with wavelengths from 3.7 to $14.5 \mu \mathrm{m}$ are the thermal emissive bands (TEB). They are calibrated on-orbit by a v-grooved BB. The on-board BB is nominally controlled at a constant temperature, $290 \mathrm{~K}$ for Terra MODIS and $285 \mathrm{~K}$ for Aqua MODIS, and periodically goes through a warn-up and cooldown (WUCD) cycle, during which the BB temperature vary from instrument ambient to up to $315 \mathrm{~K}$. The SRCA is a unique device that can be configured and operated in three different modes: radiometric, spatial, and spectral. It can track 
RSB radiometric calibration and spectral characterization stability and perform RSB and TEB spatial characterization. The SRCA radiometric mode is operated more frequently than its spatial and spectral modes.

VIIRS observations are made in 14 RSB (M1-M11 and I1-I3) covering a near-identical spectral range as MODIS RSB, 7 TEB (M12-M16 and I4-I5) with wavelengths from 3.7 to $12.4 \mu \mathrm{m}$, and 1 panchromatic DNB with a wavelength range from 0.5 to $0.9 \mu \mathrm{m}$. Table 1 provides a comparison of VIIRS and MODIS spectral bands in terms of their spectral range and nadir spatial resolution. Like MODIS, the SD/SDSM system is used for VIIRS RSB calibration. The VIIRS has a solar attenuation screen fixed in front of the SD panel but does not have an SD door to cover the SD port. Because of this, the SD calibration is performed every orbit. Over a short period at the mission beginning, the VIIRS SDSM was operated every orbit. Since then, it has been operated on a daily basis, which is still much more frequent than MODIS SDSM operation. The low gain stage of the DNB is also calibrated by the SD. VIIRS TEB calibration is provided by the on-board BB and the BB is operated exactly the same way as MODIS BB, including periodic WUCD. VIIRS does not have a device like MODIS SRCA, which is specially designed and operated for sensor on-orbit spectral and spatial characterization. Consequently, alternative approaches have to be considered. More details on MODIS and VIIRS onorbit calibration and characterization can be found in a number of references ${ }^{9-18}$.

Table 1 VIIRS and MODIS spectral bands spectral range and nadir spatial resolution (HSR: horizontal sampling resolution). VIIRS M1-M5, M7, and M13 are dual gain bands.

\begin{tabular}{|c|c|c|c|c|c|}
\hline VIRS Band & Spectral Range (um) & Nadir HSR (m) & MODIS Band(s) & Range & HSR \\
\hline DNB & $0.500-0.900$ & & & & \\
\hline O M1 & $0.402-0.422$ & 750 & 8 & $0.405-0.420$ & 1000 \\
\hline O MR & $0.436-0.454$ & 750 & 9 & $0.438-0.448$ & 1000 \\
\hline$O M B$ & $0.478-0.498$ & 750 & 3 & $\begin{array}{l}0.459-0.479 \\
0.483-0.493\end{array}$ & $\begin{array}{c}500 \\
1000\end{array}$ \\
\hline O M & $0.545-0.565$ & 750 & 4 or 12 & 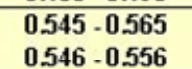 & $\begin{array}{c}500 \\
1000\end{array}$ \\
\hline 11 & $0.600-0.680$ & 375 & 1 & $0.620-0.670$ & 250 \\
\hline M.5 & $0.662-0.682$ & 750 & 13 or 14 & $\begin{array}{l}0.662-0.672 \\
0.673-0.683\end{array}$ & 1000 \\
\hline$M 6$ & $0.739-0.754$ & 750 & 15 & $0.743-0.753$ & 1000 \\
\hline R & $0.846-0.885$ & 375 & 2 & $0.841-0.876$ & 250 \\
\hline MP & $0.846-0.885$ & 750 & 16 or 2 & $\begin{array}{l}0.862-0.877 \\
0.841-0.876\end{array}$ & $\begin{array}{c}1000 \\
250\end{array}$ \\
\hline $\mathbf{4}+8$ & $1230-1250$ & 750 & 5 & SAMAE & 500 \\
\hline Ma & $1.371-1.386$ & 750 & 26 & $1.360-1.390$ & 1000 \\
\hline $\mathbf{B}$ & $1.580-1.640$ & 375 & 6 & $1.628-1.652$ & 500 \\
\hline M10 & $1580-1.640$ & 750 & 6 & $1.628-1.652$ & 500 \\
\hline M11 & $2225-2275$ & 750 & 7 & $2.105-2.155$ & 500 \\
\hline 4 & $3.550-3.930$ & 375 & 20 & $3.660-3.840$ & 1000 \\
\hline M12 & $3.660-3.840$ & 750 & 20 & SAMA & 1000 \\
\hline M13 & $3.973-4.128$ & 750 & 21 or 22 & $\begin{array}{l}3.929-3.989 \\
3.929-3.989\end{array}$ & $\begin{array}{l}1000 \\
1000\end{array}$ \\
\hline M14 & $8.400-8.700$ & 750 & 29 & SAME & 1000 \\
\hline M15 & $10263-11263$ & 750 & 31 & $10.780-11280$ & 1000 \\
\hline 5 & $10.500-12.400$ & 375 & 31 or 32 & $\begin{array}{l}10.780-11280 \\
11.770-12270\end{array}$ & $\begin{array}{l}1000 \\
1000\end{array}$ \\
\hline M16 & $11.538-12.488$ & 750 & 32 & $11.770-12270$ & 1000 \\
\hline
\end{tabular}




\section{LUNAR OBSERVATIONS}

The Moon has been used as a stable radiometric calibration and spatial characterization target by a number of earthobserving sensors. Since launch, lunar observations have been made regularly by both Terra and Aqua MODIS. MODIS lunar observations are made through its space view (SV) port. Since the lunar irradiance strongly depends on the viewing geometry, for calibration stability monitoring purposes, MODIS lunar observations have been scheduled and made at the "same" phase angles: $55^{\circ}$ to $56^{\circ}$ (waning) for Terra MODIS and $-55^{\circ}$ to $-56^{\circ}$ (waxing) for Aqua MODIS. This lunar calibration strategy is accomplished via spacecraft roll maneuvers. For MODIS, the scheduled lunar observations are made during the spacecraft nighttime. A sector rotation operation, in which the data collection time of each sector (SD, $\mathrm{SV}, \mathrm{BB}, \mathrm{SRCA}$, and EV) is shifted by a pre-determined amount, is performed during scheduled lunar calibration events. With this data collection time offset, the lunar data (images) are stored in the EV data sector. As of August, 2013, Terra and Aqua MODIS have scheduled and implemented more than 125 and 100 lunar calibration events, respectively. Table 2 is a summary of MODIS lunar calibration events on a yearly basis. Example of the lunar images from Aqua MODIS bands 1 and 2 are illustrated in Figure 2.

Table 2 Scheduled lunar observations for Terra and Aqua MODIS and SNPP VIIRS (as of August 2013)

\begin{tabular}{|c|c|c|c|c|c|c|c|c|c|c|c|c|c|c|}
\hline Year & 2000 & 2001 & 2002 & 2003 & 2004 & 2005 & 2006 & 2007 & 2008 & 2009 & 2010 & 2011 & 2012 & 2013 \\
\hline Terra MODIS & 9 & 10 & 10 & 9 & 10 & 8 & 9 & 9 & 10 & 8 & 9 & 8 & 10 & 7 \\
\hline Aqua MODIS & & & 5 & 9 & 10 & 9 & 9 & 7 & 9 & 11 & 10 & 8 & 10 & 6 \\
\hline SNPP VIIRS & & & & & & & & & & & & & 8 & 5 \\
\hline
\end{tabular}
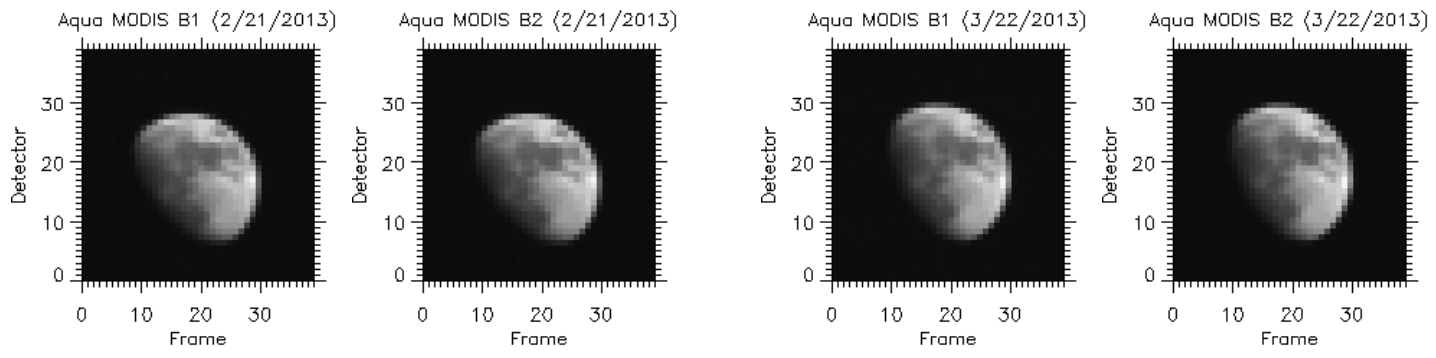

Figure 2 Lunar images from Aqua MODIS bands 1 and 2 (all detectors in a single scan).
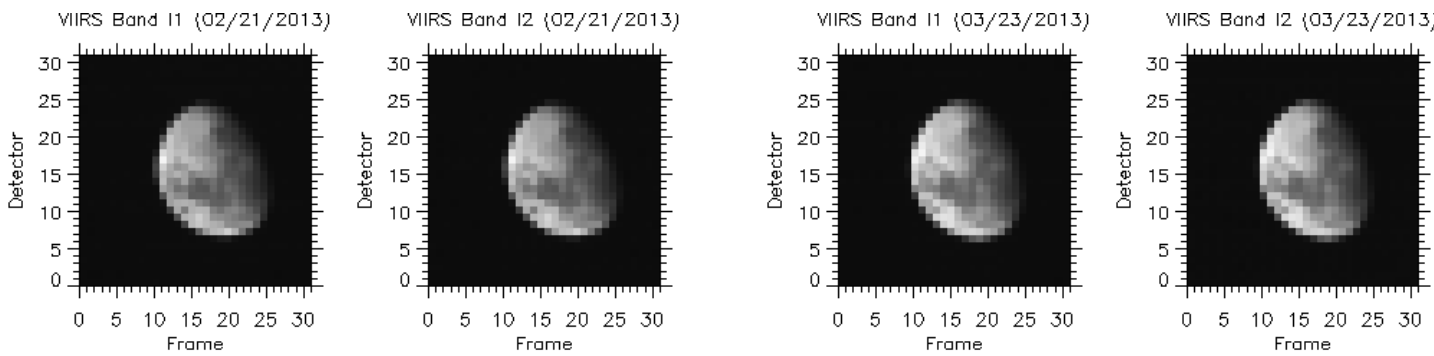

Figure 3 Lunar images from S-NPP VIIRS bands I1 and I2 (all detectors in a single scan).

Exactly the same strategies and lessons learned from MODIS lunar calibration are applied to VIIRS. The VIIRS SV port is an extension of its EV port. Because of its location (different from MODIS), VIIRS lunar observations are made during the spacecraft daytime. As shown in Table 2, VIIRS has scheduled a total of 13 lunar calibration events since launch. Due to the spacecraft operation and roll angle constraints, the VIIRS lunar observations are currently made at the 
phase angles from $-51.5^{\circ}$ to $-50.5^{\circ}$. Example of the lunar images produced by S-NPP VIIRS bands I1 and I2 are illustrated in Figure 3. Like MODIS, a sector rotation is implemented such that the EV data sector is centered at the SV. For the dual gain bands, the detector gains are fixed at high gains during scheduled lunar observations. Unlike MODIS, the data from VIIRS calibration sectors (SD, SV, and BB) are not co-registered. With the sector rotation, lunar data are collected in the EV sector and thus the images from all spectral bands are co-registered.

In preparation and support of MODIS on-orbit lunar calibration, a special scheduling tool was developed by the MODIS Characterization Support Team (MCST). Parameters, such as lunar calibration orbit/time, lunar phase and libration angles, and spacecraft roll angles, are accurately determined and provided to the Flight Operation Team (FOT) before each scheduled lunar calibration event. The same tool was also adopted to support VIIRS lunar calibration. For independent verification purpose, a separate lunar planner tool was also developed by the VIIRS Characterization Support Team (VCST). In addition to regularly scheduled lunar observations at the same phase angles, there are, from time to time, un-scheduled lunar images at different phase angles crossing through the SV port of both MODIS and VIIRS instruments.

\section{APPLICATIONS AND RESULTS}

MODIS and VIIRS lunar observations have been extensively used to support their on-orbit calibration and characterization. This section provides a brief description of methodologies developed for various applications of MODIS lunar observations. Currently, these methodologies have also been applied to VIIRS lunar observations. Specific applications and results discussed in the section include radiometric stability monitoring for RSB and TEB, spatial characterization for sensor BBR and MTF, optical leak and crosstalk assessment via lunar observations, and sensor calibration inter-comparison. More details on each of the topics presented here can also be found in a number of references ${ }^{19-27}$.

\subsection{Radiometric Calibration Stability - RSB}

MODIS RSB on-orbit calibration coefficients, $\left(\mathrm{m}_{1}\right)_{\mathrm{SD}}$, are derived from detector responses to the SD for each band, detector, sub-sample, and mirror side using the following expression,

$$
\left(m_{1}\right)_{S D}=F_{S D} /\left(d n^{*}\right)_{S D}
$$

where $\mathrm{F}_{\mathrm{SD}}$ is a reference parameter that depends on the SD BRF, its on-orbit degradation, the SD screen transmission, solar illumination and viewing angles, and the Earth-Sun distance. $\left(\mathrm{dn}^{*}\right)_{\mathrm{SD}}$ is detector response corrected for instrument background, mirror scan angle, and temperature effect. Compared to SD, the lunar surface reflectance properties are known to be extremely stable. Because of this, MODIS lunar observations have been used to track the RSB calibration stability. With lunar observations, Eqn. 1 becomes,

$$
\left(m_{1}\right)_{\text {Moon }}=F_{\text {Moon }} /(d n *)_{\text {Moon }} \text {. }
$$

In this case, the reference factor, $\mathrm{F}_{\mathrm{Moon}}$, depends on the lunar surface reflectance properties, which vary with the lunar viewing geometry, and the Earth-Moon and Moon-Sun distances. A lunar model, such as the one developed by the USGS Robotic Lunar Observatory (ROLO) program can be used to derive the reference factor ${ }^{28}$.

Figure 4 shows Aqua MODIS RSB normalized gains $\left(1 / \mathrm{m}_{1}\right)$ derived from SD and lunar observations for a number of VIS and NIR spectral bands. Though the gain profiles from both SD and lunar observations show similar trends, the magnitudes of the gain changes are not the same depending on the spectral band wavelengths. This is because MODIS SD and lunar observations are made at different angle of incidence (AOI) to the scan mirror. For both Terra and Aqua MODIS, lunar observations have been used together with SD and EV observations at different AOIs to derive and update sensor time-dependent response versus scan-angle (RVS). 

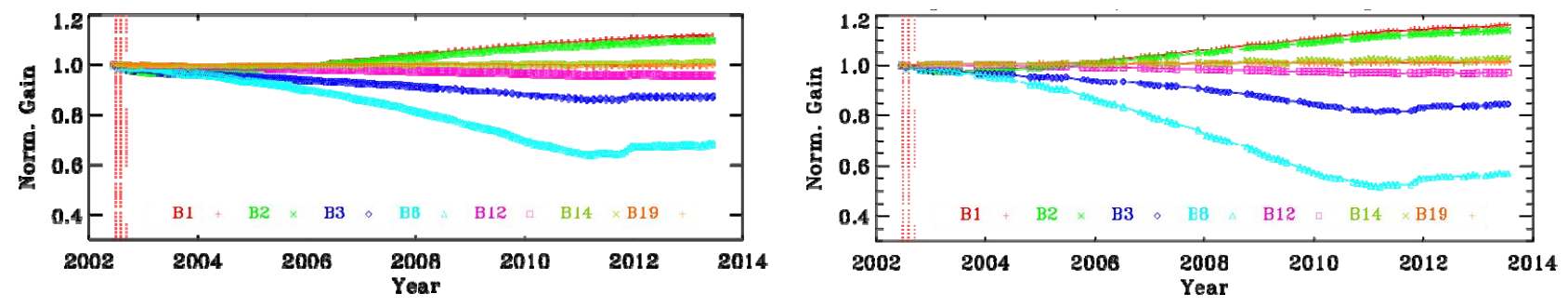

Figure 4 Aqua MODIS RSB normalized gains (bands 1, 2, 3, 8, 12, 14, and 19) derived from SD observations (left) and lunar observations (right). Results are band averaged from mirror side 1 .
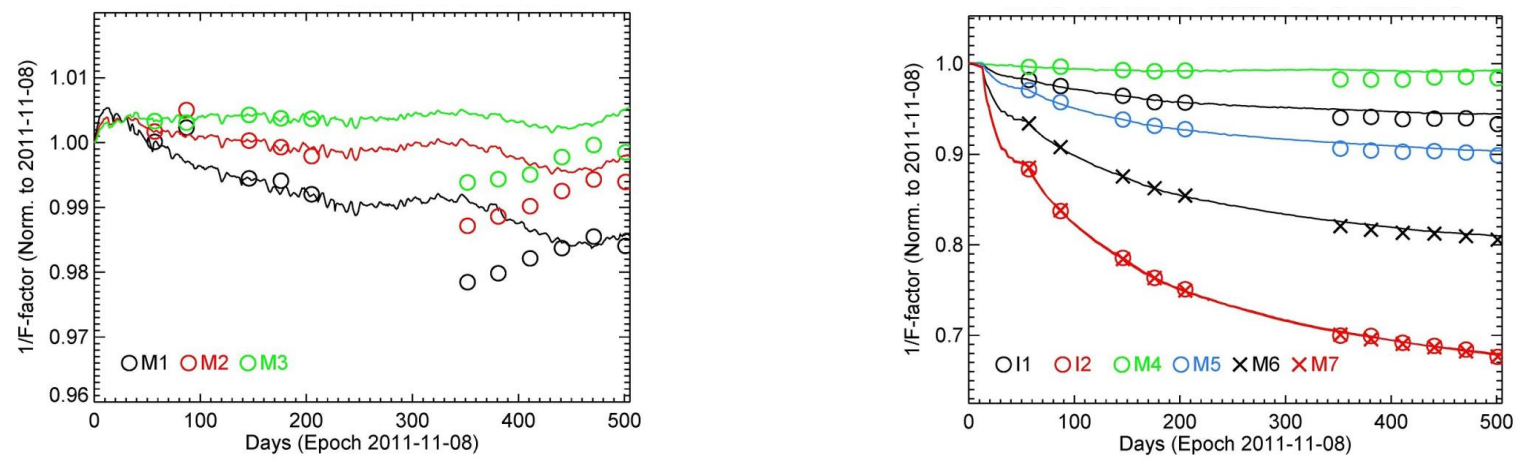

Figure 5 S-NPP VIIRS VIS/NIR band normalized gains derived from SD observations (solid curves) and lunar observations (symbols). Results are band averaged for the high gains and HAM side A.

VIIRS RSB on-orbit calibration coefficients are called the F-factors, which are inversely proportional to the detector gains. Unlike MODIS, VIIRS lunar observations through its SV port are made at the same AOI as SD observations. Therefore, the F-factors derived from lunar observations can be used to independently validate the F-factors derived from SD observations. Figure 5 shows examples of normalized gains (1/F-factors) derived from SD (solid curves) and lunar (symbols) observations for the VIIRS VIS/NIR bands. The gains in Figure 5 are band averaged (high gains for the dual gain bands) from side $\mathrm{A}$ of the half angle mirror (HAM). As expected, the results derived from SD calibration agree well with that derived from lunar observations, except for bands M1-M3. The causes for the small differences in M1-M3 are yet to be determined.

VIIRS DNB has three different gain stages, enabling observations to be made over a very large dynamic range. The SD observations can be used for the DNB low gain calibration. The data collected before and after full illumination of the Sun onto the SD can be used to determine the gain ratios of high to medium gains and medium to low gains. During scheduled lunar observations, the DNB collects data in auto gain mode. Although the same lunar calibration strategy is applied to DNB, special effort needs to be made to analyze and process the DNB lunar observations. Preliminary progress has been made recently by VCST to explore the use of on-orbit lunar observations to independently monitor the DNB radiometric calibration stability.

\subsection{Radiometric Calibration Stability - TEB}

Regularly scheduled lunar observations can also be used to monitor TEB long-term radiometric calibration stability since they are made at the same phase angles that repeat ver time. For earth-observing sensors, most of the TEB (detectors) saturate when viewing the Moon. Only a few low gain bands, e.g. MODIS band 21, may not saturate. For the bands that do not saturate, the brightness temperature (BT) can be computed for each of the lunar pixels. Since the BT is retrieved using calibration coefficients derived from instrument on-board BB, the time series of the BT, after correction for the viewing geometry differences, can be used to assess the TEB (or on-board BB) calibration stability. For the bands with 
saturated lunar pixels, a simple relative approach has been developed to track the calibration stability. This approach examines the long-term drift of the BT differences between non-saturated pixels (in the saturated band) and spatially matched or geo-located pixels in a reference non-saturated band. Results derived from this method are illustrated in Figure 6 for Terra MODIS bands 32 and 33 using band 31 as the reference. The trending is on a detector by detector basis. Different colors denote different detectors. Due to wavelength dependent lunar surface emissivity, the BT differences between the two spectral bands are not important as long as there is no drift in the BT difference time series. This approach has also been used to monitor Aqua MODIS and S-NPP VIIRS TEB calibration stability.
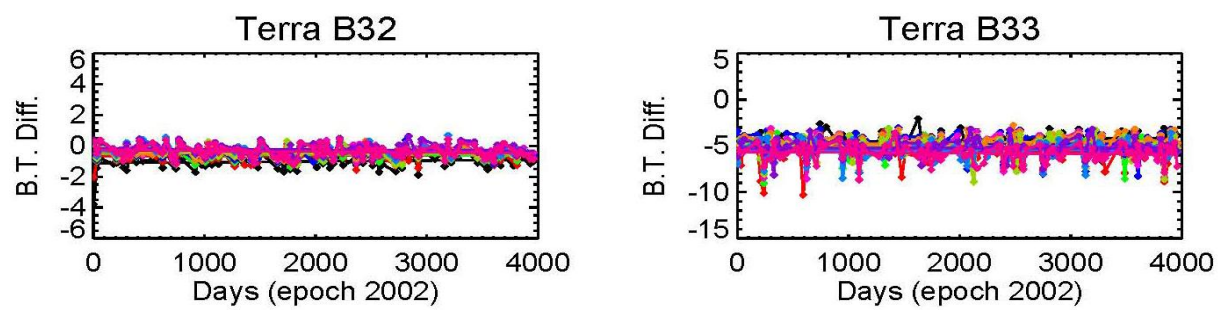

Figure 6 Terra MODIS TEB lunar response trending in brightness temperature (BT) for bands 32 (left) and 33 (right)

\subsection{Spatial Characterization - BBR}

Several approaches have been developed by MCST to characterize MODIS BBR using its lunar observations ${ }^{25-26}$. One of the methods is to compute the centroid for each detector using its lunar responses in both the along scan and along track directions. The centroid difference between two detectors is a measure of their detector-to-detector registration (DDR). The BBR is determined from band averaged centroid over all detectors. In the along-scan direction, a correction for the detector spatial resolution, thus detector size and number of sub-samples, is needed. In the along-track direction, a lunar over-sampling factor, which is related to the number of scans each detector views the Moon, should also be considered. The BBR characterization approaches developed from MODIS have been applied to VIIRS ${ }^{29}$. Presented in Figure 7 are the VIIRS RSB BBR results in both along-scan and along-track directions, derived from its on-orbit lunar observations using the centroid approach described above.
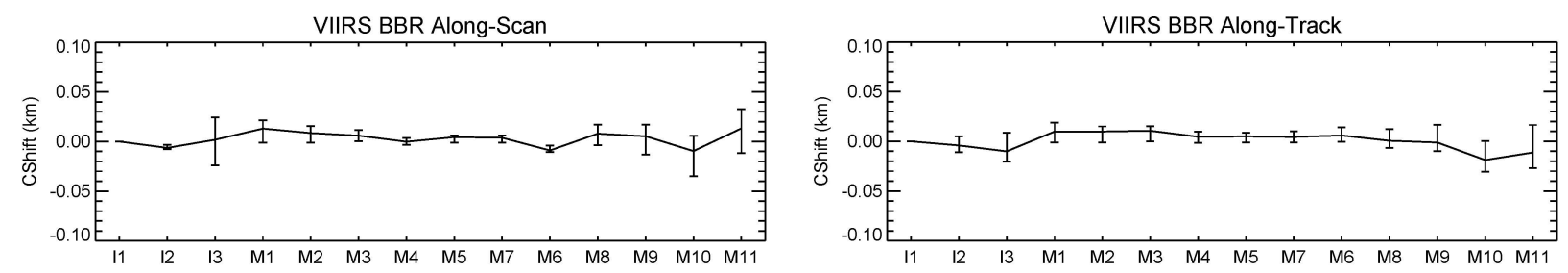

Figure 7 VIIRS RSB along-scan and along-track BBR results derived from its lunar observations.

\subsection{Spatial Characterization - MTF}

The MTF is another key spatial characterization parameter, which is often used to quantify sensor's image quality. Different methodologies have been developed using lunar observations to compute the sensor along-scan and along track $\mathrm{MTF}^{30-31}$. These methods have been applied to both MODIS and VIIRS. Because the lunar surface is not radiometrically uniform, the key to the lunar MTF characterization is to construct an edge response function (ERF) from lunar observations. The MTF is determined from the line spread function (LSF), which is differential of the ERF. For the moderate resolution imaging sensors, such as MODIS and VIIRS, it is more difficult to derive the MTF in the along-scan direction due insufficient data samples and limited movement of lunar images along this direction. Examples in Figure 8 are the on-orbit along-track MTF trends for Terra MODIS bands 1 and 3. 

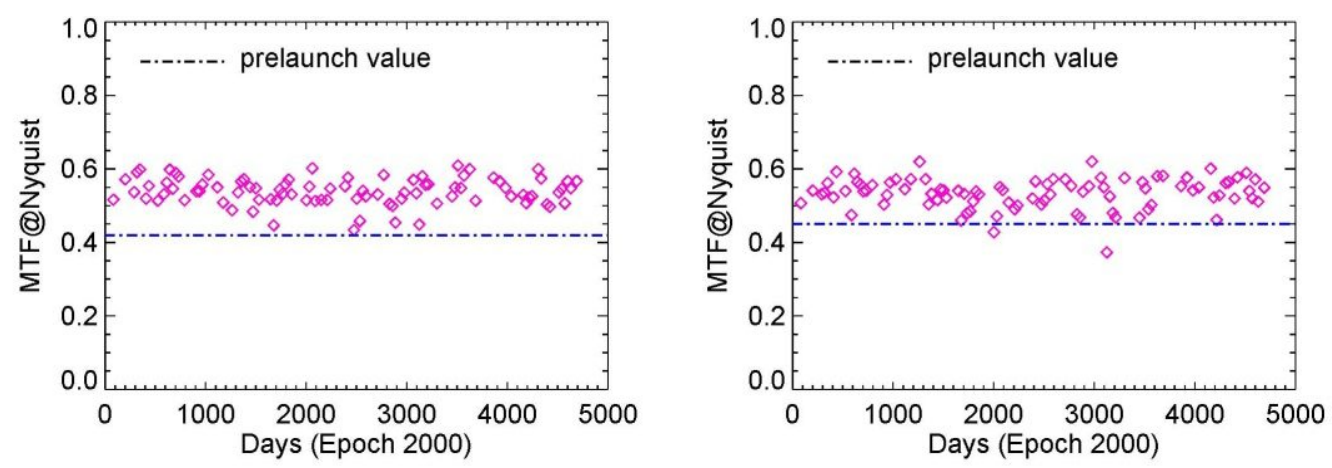

Figure 8 Along-track MTF trends derived from lunar observations for Terra MODIS bands 1 and 3 (at Nyquist frequency).

\subsection{Sensor Optical Leak and Electronic Crosstalk Characterization}

As a finite calibration target, the Moon can be used to characterize sensor optical leak and/or electronic crosstalk. This is because not all the bands/detectors view the lunar surface at the same time. Therefore, it is relatively easy to establish the relationship between the sending and receiving bands. For Terra MODIS, lunar observations have been to determine and quantify the optical leak from its band 31 to other photoconductive (PC) bands (32-36) on the LWIR focal plane assembly (FPA). The optical leak correction coefficients derived from lunar observations have been used in the L1B algorithm $^{27}$. Both Terra and Aqua MODIS also used the lunar observations under different operational conditions at the mission beginning to assess the electronic crosstalk among different spectral bands on the SMIR FPA and its calibration impact.

At launch, the electronic crosstalk in Terra MODIS band 27 was very small. Regularly scheduled lunar observations have found that its electronic crosstalk has gradually increased since launch. Shown in Figure 9 are the lunar images from multiple scans of band 27 detector 1 . As expected, there are many saturated pixels at the center of the lunar image. The electronic crosstalk is evident in the areas outside of the expected lunar responses. The electronic crosstalk generally had a positive response in 2002 while it has become predominantly negative in 2010 . The location of the electronic crosstalk can be used to determine where the crosstalk comes from or which sending band/detector is responsible. From these lunar images, crosstalk correction coefficients can be derived and possibly used for the correction algorithm ${ }^{32}$. From VIIRS lunar images collected at mission beginning, it was found that the optical leak and electronic crosstalk in most of its spectral bands were extremely small and their calibration impact can be ignored.
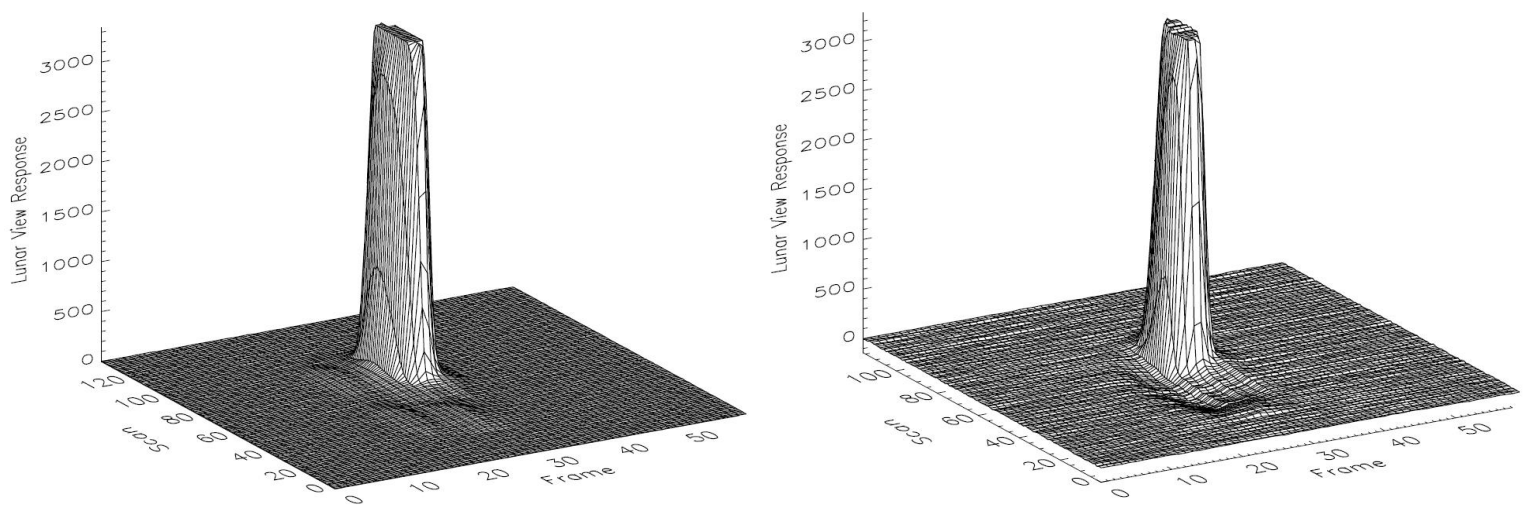

Figure 9 Lunar images observed from Terra MODIS band 27 (detector 1, multiple scans) on January 3, 2002 (left) and on January 4, 2010 (right). 


\subsection{Calibration Inter-comparison}

One of the most important applications for lunar observations is allowing calibration inter-comparison to be made among different sensors. These sensors could be of different types, operated on different platforms, and acquiring lunar measurements at different times. Differences in sensor spectral response and lunar viewing geometry can be corrected by referencing to a common lunar model via the following expression,

$$
R=\left(\mathrm{I}_{\mathrm{A}_{-} \text {Meas }} / \mathrm{I}_{\mathrm{A}_{-} \text {Model }}\right) /\left(\mathrm{I}_{\mathrm{B}_{-} \text {Meas }} / \mathrm{I}_{\mathrm{B}_{-} \text {Model }}\right) \text {. }
$$

where $\mathrm{I}_{\mathrm{A} \_ \text {Meas }}$ and $\mathrm{I}_{\mathrm{B} \_ \text {Meas }}$ are the measured lunar irradiances by sensor $\mathrm{A}$ and $\mathrm{B}$ and $\mathrm{I}_{\mathrm{A} \_ \text {Model }}$ and $\mathrm{I}_{\mathrm{B} \_ \text {Model }}$ are the predicated lunar irradiances by a common lunar model. Figure 10 presents the time series for the ratios of sensor (MODIS and VIIRS) measured lunar irradiance to the ROLO model predicated lunar irradiance. Noticeable variations in the time series are likely due to the measurement errors and non-perfect corrections for the lunar viewing geometry. The ratio value itself is not important for calibration inter-comparison purposes. The ratio difference between a pair of sensors is likely due to their calibration, and/or calibration traceability difference, and possibly their solar irradiance model difference.
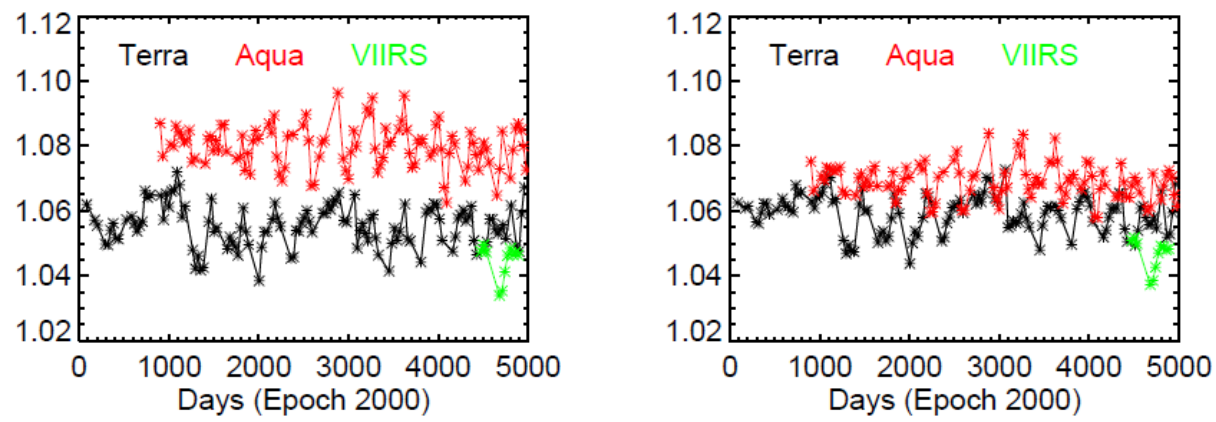

Figure 10 Ratios of MODIS and VIIRS lunar irradiance (measured) to ROLO model predicated lunar irradiance (left: MODIS band 8 and VIIRS M1; right: MODIS band 9 and VIIRS M2).

MCST has used lunar observations to compare Terra and Aqua MODIS calibration consistency and to cross-calibrate with other sensors ${ }^{21}$. The same strategy is currently applied to inter-compare VIIRS with other sensors.

\section{SUMMARY}

This paper provides a brief description of MODIS and VIIRS on-board calibration and characterization activities, including their regularly scheduled lunar observations. It describes different methodologies developed to use the Moon to support sensor on-orbit calibration and characterization. Applications and results presented in this paper include using the Moon to track MODIS and VIIRS RSB and TEB calibration stability and to assess sensor spatial performance in terms of BBR and MTF parameters. In addition, MODIS and VIIRS lunar observations have been used to characterize sensor optical leak and electronic crosstalk and associated calibration impact. Most of the methodologies, initially develop for MODIS lunar observations, are now adopted for VIIRS lunar observations and applications. It is expected that theses methodologies can also be applied to other earth-observing sensors.

\section{ACKNOWLEDGEMENTS}

The authors would like to thank other members of the MODIS Characterization Support Team (MCST) and VIIRS Characterization Support Team (VCST) for their technical assistance and support. 


\section{REFERENCES}

1. W.L. Barnes and V.V. Salomonson, "MODIS: A global image spectroradiometer for the Earth Observing System," Critical Reviews of Optical Science and Technology, CR47, 285-307, 1993

2. W.L. Barnes, X. Xiong, and V.V. Salomonson, "Status of Terra MODIS and Aqua MODIS," J. of Advances in Space Research, 32/11, 2099-2106, 2003

3. X. Xiong, K. Chiang, J. Esposito, B. Guenther, and W.L. Barnes, "MODIS On-orbit Calibration and Characterization," Metrologia 40 89-92, 2003

4. Salomonson V.V., W.L. Barnes, X. Xiong, S. Kempler, and E. Masuoka, "An Overview of the Earth Observing System MODIS Instrument and Associated Data Systems Performance," Proceedings of IGARSS 2002

5. Ardanuy, P., C. F. Schueler, S. W. Miller, P. S. Kealy, S. A. Cota, J. K. Haas, and C. Welsch, "NPOESS VIIRS Design Process," Proc. SPIE, vol. 4483, 22-34, 2002

6. Schueler, C. F., E. Clement, P. Ardanuy, C. Welsh, F. De Luccia, and H. Swenson, "NPOESS VIIRS sensor design overview," Proc. SPIE, vol. 4483, 11-23, 2002

7. Murphy, R.P., P. E. Ardanuy, F. Deluccia, J. E. Clement, and C. Schueler, "The visible infrared imaging radiometer suite, Earth Science Satellite Remote Sensing,” vol. 1, New York, USA: Springer-Verlag, pp. 199-223, 2006

8. Cao, C., F. Deluccia, X. Xiong, R. Wolfe, and F. Weng, "Early On-orbit Performance of the Visible Infrared Imaging Radiometer Suite (VIIRS) onboard the Suomi National Polar-orbiting Partnership (S-NPP) Satellite," IEEE Trans. Geosci. Remote Sens. (in press)

9. Xiong X., J. Sun, W. Barnes, V. Salomonson, J. Esposito, H. Erives, and B. Guenther, "Multi-year On-orbit Calibration and Performance of Terra MODIS Reflective Solar Bands," IEEE Transactions on Geoscience and Remote Sensing, Vol. 45, No. 4, 879-889, 2007

10. X. Xiong, J. Sun, X. Xie, W. Barnes, and V. Salomonson, "On-Orbit Calibration and Performance of Aqua MODIS Reflective Solar Bands," IEEE Trans. Geosci. Remote Sens., 48(1), 535-546, 2010

11. Xiong X, K. Chiang, A. Wu, W.L. Barnes, B. Guenther, and V. Salomonson, "Multiyear On-orbit Calibration and Performance of Terra MODIS Thermal Emissive Bands," IEEE Transactions on Geoscience and Remote Sensing, $46(6), 1790-1803,2008$

12. Xiong X., B. N. Wenny, A. Wu, W. Barnes, and V. Salomonson, "Aqua MODIS Thermal Emissive Bands On-orbit Calibration, Characterization, and Performance," IEEE Trans. Geosci. Remote Sens., 47(3), 803-814, 2009

13. Xiong X, N. Che, and W.L. Barnes, "Terra MODIS On-orbit Spatial Characterization and Performance," IEEE Transactions on Geoscience and Remote Sensing, Vol. 43, No. 2, 355-365, 2005

14. Xiong X, N. Che, and W.L. Barnes, "Terra MODIS On-orbit Spectral Characterization and Performance", IEEE Transactions on Geoscience and Remote Sensing, Vol. 44, No. 8, 2198-2206, 2006

15. Fulbright, J., N. Lei, K. Chiang, X. Xiong, "Characterization and performance of the Suomi-NPP/VIIRS solar diffuser stability monitor," Proc. SPIE 8510, 851015, 2012

16. Oudrari, H., J. McIntire, D. Moyer, K. Chiang, X. Xiong, and J. Butler, Preliminary assessment of Suomi-NPP VIIRS on-orbit radiometric performance, Proc. SPIE, vol. 8510, 851011, 2012

17. Lei, N., Z. Wang, J. Fulbright., S. Lee, J. McIntire, K. Chiang, and X. Xiong, "Initial on-orbit radiometric calibration of the Suomi NPP VIIRS Reflective Solar Bands," Proc. SPIE, vol. 8510, 851018, 2012

18. Xiong, X., J. Butler, K. Chiang, B. Efremova, J. Fulbright, N. Lei, J. McIntire, H. Oudrari, J. Sun, Z. Wang, and A. Wu, "VIIRS On-orbit Calibration Methodology and Performance," JGR (submitted)

19. Barnes WL, Xiong X, Eplee R, Sun J, and Lyu CH, "Use of the Moon for Calibration and Characterization of MODIS, SeaWiFS, and VIRS," Earth Science Satellite Remote Sensing: Data, Computational Processing, and Tools, Vol. 2, Chapter 6, 98-119, Springer, 2006

20. Sun J, X. Xiong, W. Barnes, and B. Guenther, "MODIS Reflective Solar Bands On-orbit Lunar Calibration," accepted for IEEE Transactions on Geoscience and Remote Sensing, Vol. 45, No. 7, 2383-2393, 2007

21. Xiong, X., J. Sun, and W. Barnes, "Inter-comparison of On-orbit Calibration Consistency between Terra and Aqua MODIS Reflective Solar Bands Using the Moon," IEEE Geosci. Remote Sens. Let., 5(4), 778-782, 2008

22. Eplee, R.E. Jr., J. Sun, G. Meister, F.S. Patt, X. Xiong, and C.R. McClain, "Cross calibration of SeaWiFS and MODIS using on-orbit observations of the Moon," Appl. Opt. 50, 120-133, 2011

23. Sun, J., X. Xiong, and J. Butler, "NPP VIIRS on-orbit calibration and characterization using the moon," Proc. SPIE, vol 8510, 85101I, 2012

24. X. Xiong, J. Sun, K. Chiang, S. Xiong, and W.L. Barnes, "MODIS On-orbit Characterization Using the Moon," 
Proceedings of SPIE - Sensors, Systems, and Next Generation Satellite VI, 4881, 299-307, 2003

25. X. Xiong, J. Sun, S. Xiong, and W.L. Barnes, "Using the Moon for MODIS On-orbit Spatial Characterization", Proceedings of SPIE - Sensors, Systems, and Next Generation of Satellites VII, 5234, 480-487, 2004

26. Xiong, X., J. Sun, A. Angal, K. Chiang, and W. Barnes, "Applications and results of MODIS lunar observations, Sensors, Systems, and Next Generation Satellites XI", Proc. SPIE, vol. 6744, no. 67441H, 2007

27. Li, W., X. Xiong, K. Chiang, and G. Toller, "Evaluation of Terra MODIS PC bands optical leak correction algorithm, Earth Observing Systems X", Proc. SPIE, vol. 5882, no. 588219, 2005

28. Stone, T. and H. Kieffer, "An absolute irradiance of the Moon for on-orbit calibration", Proceedings of SPIE - Earth Observing Systems VII, 4814, 211-221, 2002

29. Wang, Z. and X. Xiong, "On-orbit spatial characterization of VIIRS using the Moon," Proc. SPIE, vol 8866, 2013

30. Choi, T., X. Xiong, Z Wang, "On-Orbit Lunar Modulation Transfer Function (MTF) Measurements for the Moderate Resolution Imaging Spectroradiometer (MODIS)," IEEE Transactions on Geoscience and Remote Sensing (accepted)

31. Wang, Z., X. Xiong, T. Choi, and D. Link, "On-orbit Characterization of MODIS Modulation Transfer Function Using the Moon," IEEE Transactions on Geoscience and Remote Sensing, (accepted)

32. Sun, J., X. Xiong, S. Madhavan, and B. Wenny, "Terra MODIS Band 27 Electronic Crosstalk Effect and Its Removal," IEEE Transactions on Geoscience and Remote Sensing (accepted) 